\title{
Efficacy and toxicity of nimotuzumab combined with radiotherapy in elderly patients with esophageal squamous cell carcinoma
}

\author{
JIN-HUA GUO*, MING-QIU CHEN*, CHENG CHEN, HAI-JIE LU and BEN-HUA XU \\ Department of Radiation Oncology, Fujian Medical University Union Hospital, Fuzhou, Fujian 350001, P.R. China
}

Received June 3, 2015; Accepted June 27, 2015

DOI: $10.3892 / \operatorname{mco} .2015 .606$

\begin{abstract}
This study was conducted to assess the efficacy and toxicity of nimotuzumab combined with radiotherapy (RT) in elderly patients with esophageal squamous cell carcinoma. The clinical data of 16 esophageal squamous cell carcinoma patients, aged $>70$ years, who were initially treated with nimotuzumab combined with RT, were collected and retrospectively reviewed. The overall response and treatment toxicity were analyzed using SPSS software. All the patients completed the treatment schedule. The response to treatment was assessed at treatment completion and reassessed after 1-2 months: 1 patient achieved complete response (CR), 10 patients achieved partial response (PR), 4 patients exhibited stable disease and 1 patient developed disease progression and succumbed to radiation pneumonitis (RP) 1 month later. The overall response rate (CR+PR) was $68.8 \%$. All 16 patients experienced grade 1-2 radiation esophagitis; no grade 3-4 toxicities were reported. There was one case of treatment-related mortality due to RP during the study. One patient developed a rash on the forearm. No hematological, gastrointestinal, hepatic or renal toxicities were observed. In conclusion, the toxicity of combined nimotuzumab with RT in elderly patients with esophageal cancer was tolerable. However, due to limitations associated with the retrospective nature of this study, the limited number of enrolled cases and the epidermal growth factor receptor expression determination prior to treatment, the efficacy of this treatment modality requires further investigation.
\end{abstract}

Correspondence to: Professor Ben-Hua Xu, Department of Radiation Oncology, Fujian Medical University Union Hospital, 29 Xinquan Road, Fuzhou, Fujian 350001, P.R. China

E-mail: benhuaxu@163.com

*Contributed equally

Key words: nimotuzumab, anti-epidermal growth factor receptor antibody, radiotherapy, elderly, esophageal squamous cell carcinoma

\section{Introduction}

Esophageal cancer (squamous cell carcinoma or adenocarcinoma) is a highly lethal disease, with the 5-year survival rate rarely exceeding $25 \%$ (1). Esophageal carcinoma is rare among young individuals and increases in incidence with age, peaking in the seventh and eighth decades of life (2). With the increase in the aging general population, the number of elderly patients with esophageal cancer is expected to increase in the foreseeable future.

Surgery is currently the mainstay of treatment for esophageal cancer. However, due to the limitations of the physiological conditions and the relatively high rates of postoperative morbidity and mortality, elderly patients with esophageal cancer are generally not considered fit for oesophagectomy (3), with 75 years of age often being considered as the age limit for surgical intervention (4).

For patients unfit for surgery, concurrent chemoradiotherapy (CRT) is considered to be the optimal treatment. However, due to the significant toxicity of CRT, the majority of elderly patients are unable to complete the treatment schedule. Therefore, novel and efficient protocols with tolerable toxicity profiles are required for elderly patients with esophageal cancer.

Over the last decade, several studies on molecular-targeted agents, mainly tyrosine-kinase inhibitors or monoclonal antibodies, were undertaken for several cancer types and were found to be associated with acceptable toxicities (5-9), while achieving a significant survival benefit. Among those agents, the anti-epidermal growth factor receptor (EGFR) monoclonal antibodies, including panitumumab and cetuximab, were the first to be developed and widely used in the clinical setting for the treatment of rectal, breast, or gastric cancer.

Nimotuzumab, a recombinant humanized monoclonal immunoglobulin G1 antibody against human EGFR (HER-1), which blocks the binding of EGF and transforming growth factor- $\alpha$ to EGFR, were found to be promising for the treatment of tumors of epithelial origin (10). The combination of nimotuzumab with chemoradiation or RT alone was found to be safe and tolerable in two clinical studies that reported encouraging survival results in esophageal cancer $(11,12)$. However, for elderly esophageal cancer patients, who differ significantly from younger patients (13), such studies are scarce $(14,15)$. 
The present study is a retrospective investigation assessing the efficacy and toxicity of nimotuzumab combined with RT in elderly patients with esophageal squamous cell carcinoma.

\section{Patients and methods}

Pretreatment work-up and eligibility criteria. This retrospective analysis was approved by the Institutional Review Board of Fujian Medical University Union Hospital. All the information was anonymized and de-identified prior to analysis.

The pretreatment work-up included medical history, assessment of swallowing function, physical examination, standard laboratory tests, esophagogastroduodenoscopy, barium esophagography, cervical and abdominal ultrasound, chest computed tomography (CT), bone scan and magnetic resonance imaging. Bronchoscopy was performed if considered necessary. The clinical tumor-node-metastasis (cTNM) stage was determined according to the 7th American Joint Committee on Cancer TNM staging system (16) according to the CT scan findings (17).

The eligibility criteria for this retrospective study were as follows: i) Patients aged $\geq 70$ years, with histologically proven squamous cell carcinoma of the esophagus and an Eastern Cooperative Oncology Group performance status of $\leq 2$; ii) adequate bone marrow, hepatic and renal function (18); and iii) patients' written informed consent prior to treatment.

The exclusion criteria included the following: i) History of other tumors; ii) previous treatment for esophagobronchial or esophagomediastinal fistulas; and iii) other concomitant medical conditions requiring treatment.

Treatment. A total of 10 patients received $200 \mathrm{mg}$ of nimotuzumab weekly concurrently with RT, whereas the remaining 6 patients received $400 \mathrm{mg}$ nimotuzumab (diluted in $250 \mathrm{ml}$ $0.9 \%$ sodium chloride, administered as an intravenous infusion over $1 \mathrm{~h}$ ) 1 week prior to the initiation of RT, followed by $200 \mathrm{mg}$ once weekly concurrently with RT $(19,20)$.

The patients underwent three-dimensional conformal or intensity-modulated RT. The dose and target of RT and the dose limitations of the organs at risk (OARs) were previously reported (21-24). If any of the OAR dose limitations could not be satisfied, RT was defined as palliative treatment and was applied to the gross tumor only. Treatment was continued until disease progression, development of unacceptable toxicity, or withdrawal of consent.

Criteria for toxicity and treatment response. The toxicities were graded using the National Cancer Institute common toxicity criteria, version 3.0 (25). The acute RT toxicities were assessed according to the guidelines of the Radiation Therapy Oncology Group (26).

With the exception of disease progression, response was evaluated by CT scanning and barium esophagography at treatment completion and confirmed $\geq 4$ weeks later. The clinical criteria for response were defined according to the Response Evaluation Criteria in Solid Tumors, version 1.1 (27), based on the findings of CT scanning and barium esophagography. Two-dimensionally measurable lesions were defined by the length and width of the primary tumor and the diameter of the largest positive lymph node.
Table I. Clinical characteristics of the patients $(n=16)$.

\begin{tabular}{|c|c|}
\hline Characteristics & No. \\
\hline \multicolumn{2}{|l|}{ Age, years } \\
\hline Median & 77.3 \\
\hline Range & $70-84$ \\
\hline \multicolumn{2}{|l|}{ Gender } \\
\hline Male & 13 \\
\hline Female & 3 \\
\hline \multicolumn{2}{|c|}{ ECOG performance status } \\
\hline 0 & 2 \\
\hline 1 & 10 \\
\hline 2 & 4 \\
\hline \multicolumn{2}{|l|}{ Tumor location } \\
\hline Cervical & 3 \\
\hline Upper & 4 \\
\hline Middle & 8 \\
\hline Lower & 1 \\
\hline \multicolumn{2}{|l|}{ Clinical stage } \\
\hline II & 2 \\
\hline III & 12 \\
\hline $\mathrm{IVa}$ & 2 \\
\hline \multicolumn{2}{|l|}{ Treatment } \\
\hline 200 mg weekly & 10 \\
\hline 400 mg first week ${ }^{\mathrm{a}}$ & 6 \\
\hline
\end{tabular}

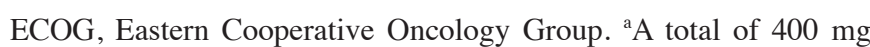
1 week prior to the initiation of RT, followed by $200 \mathrm{mg}$ weekly with concurrent RT. RT, radiotherapy.

Follow-up and statistical analysis. The patients were evaluated every 3 months for the first 2 years after treatment, every 6 months for the next 3 years and annually thereafter. Surveillance included interim history and physical examination, assessment of swallowing function, performance status and weight measurement, and laboratory testing. Esophagogastroduodenoscopy and CT of the chest and abdomen were performed every 6 months for the first 2 years and annually thereafter.

All the patient outcomes were evaluated in December, 2014. The primary endpoints were overall response and toxicity. The data were analyzed using SPSS software, version 17.0 (SPSS Inc., Chicago, IL, USA).

\section{Results}

Patient characteristics. Between October 1, 2009 and July 31, 2014, a total of 16 patients were enrolled in this study. The clinical characteristics of the patients are summarized in Table I.

All the patients succesfully completed the treatment schedule. At treatment completion, 1 patient had achieved clinical complete response (CR) in the primary tumor as well as the metastatic nodes, 1 patient experienced primary tumor CR and partial response (PR) in the metastatic nodes, 
Table II. Response to treatment.

\begin{tabular}{lcc}
\hline Response & $\begin{array}{c}1 \mathrm{st}^{\mathrm{a}} \text { assessment, } \\
\text { no. }\end{array}$ & $\begin{array}{c}2 \mathrm{nd}^{\mathrm{b}} \text { assessment, } \\
\text { no. }\end{array}$ \\
\hline CR & 1 & 1 \\
PR & 7 & 10 \\
SD & 8 & 4 \\
PD & 0 & 1 \\
Total & 16 & 16 \\
\hline
\end{tabular}

${ }^{\mathrm{a} A t}$ RT completion. ${ }^{\mathrm{b}}$ Four weeks after RT completion. CR, complete response; $\mathrm{PR}$, partial response; $\mathrm{SD}$, stable disease; $\mathrm{PD}$, progressive disease; RT, radiotherapy.

6 patients achieved PR in the primary and metastatic lesion, 8 patients exhibited stable disease (SD) and no patients exhibited progressive disease (PD). When the response was reassessed at 1-2 months after the initial assessment, 2 patients had CR, 10 had PR, 3 had SD and 1 patient developed PD and succumbed to radiation pneumonitis (RP) 1 month later. The overall rate of response to treatment is presented in Table II.

Treatment-related toxicity. All 16 patients experienced grade 1-2 radiation esophagitis (RE); no grade 3-4 toxicities were reported. One patient succumbed to RP during the study. One patient developed a rash on the forearm. No hematological, gastrointestinal, hepatic or renal toxicities were observed.

\section{Discussion}

Due to the limitations associated with the underlying medical conditions and the mean life expectancy, elderly patients with esophageal cancer undergo less intensive treatment, such as surgery or definitive CRT (3). The treatment commonly includes palliative esophageal stent implantation, improvement of symptoms by palliative chemotherapy or RT, or best supportive care. However, with the development of target drugs exhibiting lower toxicity and superior efficacy, this therapeutic plan has been changing.

Of the known available target drugs, EGFR inhibitors are beneficial in the majority of patients with tumors exhibiting EGFR overexpression, exerting a prominent antitumor effect on several cancer types, such as glioma and rectal, pancreatic and breast cancer. However, for a proportion of esophageal cancers harboring EGFR mutations, ranging between 30 and 90\% (28), in contrast to preclinical and early clinical evidence, the addition of an EGFR inhibitor to standard chemotherapy in unselected advanced early gastric cancer cases does not improve outcome, but is associated with higher toxicity rates (29). When combined with CRT or RT, the EGFR inhibitor demonstrated a survival benefit for esophageal cancer $(11,12)$. As shown in the present study, when treated with RT alone, the objective response rate was 50\% at the completion of treatment and $75 \%$ at $1-2$ months after treatment (11), with 2 patients achieving CR; the efficacy appeared to be superior to that of RT alone (30), but inferior to the historic record of CRT (31).
The most common toxicity associated with this therapy protocol was grade 1-2 RE, which was not considered to be caused by nimotuzumab, as all the patients developed RE only at RT doses of $\geq 1,800 \mathrm{cGy}$ and the symptomatic pain of acute $\mathrm{RE}$ was relieved by treatment with low-dose dexamethasone, suggesting RT-related toxicity. These results indicated that nimotuzumab exhibited lower mucosal toxicity and did not exacerbate the symptoms of RE.

One patient succumbed to acute RP 1 month after completion of treatment. The RT treatment plan was reviewed and it was found that the V5 (volume receiving an RT dose of $500 \mathrm{cGy}$ ) of the entire lung was very high (78\%), which was considered to be one most important factors leading to death.

Hematological toxicity was commonly reported in patients treated with CRT, and was the most significant issue in elderly patients with esophageal cancer. However, hematological toxicity was not common among elderly patients treated with the EGFR inhibitor. In the present study, no patient experienced any grade of hematological toxicity, including anemia, leukopenia or thrombocytopenia.

Skin toxicity, namely an acneiform rash, was the most predominant side effect and considered to be dose-related for all HER-1/EGFR-targeted agents (32-34). However, only 1 patient developed mild (grade 1) local skin rash on the forearm in the present study. This may be explained by the fact that nimotuzumab is a wholly recombinant humanized monoclonal immunoglobulin, which may result in weaker allergic reactions compared with other anti-HER-1/EGFR monoclonal antibodies, such as cetuximab and panitumumab (34).

In conclusion, when nimotuzumab was combined with RT in elderly esophageal cancer patients, the treatment-related toxicity was found to be torelable. However, due to the limitations of the present study, including its retrospective nature, the limited case number and the determination of EGFR expression prior to treatment, further investigation is required to verify the efficacy of this treatment modality.

\section{References}

1. Enzinger PC and Mayer RJ: Esophageal cancer. N Engl J Med 349: 2241-2252, 2003.

2. Rustgi AK and El-Serag HB: Esophageal carcinoma. N Engl J Med 371: 2499-2509, 2014.

3. Steyerberg EW, Neville B, Weeks JC and Earle CC: Referral patterns, treatment choices, and outcomes in locoregional esophageal cancer: A population-based analysis of elderly patients. J Clin Oncol 25: 2389-2396, 2007.

4. Kinugasa S, Tachibana M, Yoshimura H, Dhar DK, Shibakita M, Ohno S, Kubota H, Masunaga R and Nagasue N: Esophageal resection in elderly esophageal carcinoma patients: Improvement in postoperative complications. Ann Thorac Surg 71: 414-418, 2001.

5. Ferry DR, Anderson M, Beddard K, et al: A phase II study of gefitinib monotherapy in advanced esophageal adenocarcinoma: Evidence of gene expression, cellular, and clinical response. Clin Cancer Res 13: 5869-5875, 2007.

6. Rao S, Starling N, Cunningham D, et al: Matuzumab plus epirubicin, cisplatin and capecitabine (ECX) compared with epirubicin, cisplatin and capecitabine alone as first-line treatment in patients with advanced oesophago-gastric cancer: A randomised, multicentre open-label phase II study. Ann Oncol 21: 2213-2219, 2010.

7. Bang YJ, Van Cutsem E, Feyereislova A, et al; ToGA Trial Investigators: Trastuzumab in combination with chemotherapy versus chemotherapy alone for treatment of HER2-positive advanced gastric or gastro-oesophageal junction cancer (ToGA): A phase 3, open-label, randomised controlled trial. Lancet 376: 687-697, 2010 
8. Guo XF, Zhu XF, Zhong GS, Deng BG, Gao ZT and Wang H: Lapatinib, a dual inhibitor of EGFR and HER2, has synergistic effects with 5-fluorouracil on esophageal carcinoma. Oncol Rep 27: 1639-1645, 2012

9. Waddell T, Chau I, Cunningham D, et al: Epirubicin, oxaliplatin, and capecitabine with or without panitumumab for patients with previously untreated advanced oesophagogastric cancer (REAL3): A randomised, open-label phase 3 trial. Lancet Oncol 14: 481-489, 2013.

10. Ramakrishnan MS, Eswaraiah A, Crombet T, Piedra P, Saurez G, Iyer $\mathrm{H}$ and Arvind AS: Nimotuzumab, a promising therapeutic monoclonal for treatment of tumors of epithelial origin. MAbs 1: 41-48, 2009.

11. Liang J, E M, Wu G, et al: Nimotuzumab combined with radiotherapy for esophageal cancer: Preliminary study of a phase II clinical trial. Onco Targets Ther 6: 1589-1596, 2013.

12. Ma NY, Cai XW, Fu XL, Li Y, Zhou XY, Wu XH, Hu XC, Fan M, Xiang JQ, Zhang YW, et al: Safety and efficacy of nimotuzumab in combination with radiotherapy for patients with squamous cell carcinoma of the esophagus. Int J Clin Oncol 19: 297-302, 2014.

13. Takeuchi S, Ohtsu A, Doi T, Kojima T, Minashi K, Mera K, Yano T, Tahara M, Muto M and Nihei K: A retrospective study of definitive chemoradiotherapy for elderly patients with esophageal cancer. Am J Clin Oncol 30: 607-611, 2007.

14. Zhai Y, Hui ZG, Wang JB, et al: Concurrent erlotinib and radiotherapy for elderly squamous cell esophageal carcinoma: Results of a pilot study. Int J Radiat Oncol 81 (Suppl), S313, 2011.

15. Iyer R, Chhatrala R, Shefter T, et al: Erlotinib and radiation therapy for elderly patients with esophageal cancer - clinical and correlative results from a prospective multicenter phase 2 trial. Oncology 85: 53-58, 2013.

16. Rice TW, Blackstone EH and Rusch VW: 7th edition of the AJCC Cancer Staging Manual: Esophagus and esophagogastric junction. Ann Surg Oncol 17: 1721-1724, 2010.

17. Schröder W, Baldus SE, Mönig SP, Beckurts TK, Dienes HP and Hölscher AH: Lymph node staging of esophageal squamous cell carcinoma in patients with and without neoadjuvant radiochemotherapy: Histomorphologic analysis. World J Surg 26: 584-587, 2002 .

18. Gietema JA, de Vries EG, Sleijfer DT, Willemse PH, GuchelaarHJ, Uges DR, Aulenbacher P, Voegeli R and Mulder NH: A phase I study of 1,2-diamminomethyl-cyclobutane-platinum (II)-lactate (D-19466; lobaplatin) administered daily for 5 days. Br J Cancer 67: 396-401, 1993.

19. Crombet T, Osorio M, Cruz T, Roca C, del Castillo R, Mon R, Iznaga-Escobar N, Figueredo R, Koropatnick J, Renginfo E, et al: Use of the humanized anti-epidermal growth factor receptor monoclonal antibody h-R3 in combination with radiotherapy in the treatment of locally advanced head and neck cancer patients. J Clin Oncol 22: 1646-1654, 2004

20. Satoh T, Lee KH, Rha SY, et al: Randomized phase II trial of nimotuzumab plus irinotecan versus irinotecan alone as second-line therapy for patients with advanced gastric cancer. Gastric cancer: Sep 5, 2014 (Epub ahead of print).

21. Li QQ, Liu MZ, Hu YH, Liu H, He ZY and Lin HX: Definitive concomitant chemoradiotherapy with docetaxel and cisplatin in squamous esophageal carcinoma. Dis Esophagus 23: 253-259, 2010.
22. Gagliardi G, Constine LS, Moiseenko V, Correa C, Pierce LJ, Allen AM and Marks LB: Radiation dose-volume effects in the heart. Int J Radiat Oncol Biol Phys 76 (Suppl 3): S77-S85, 2010.

23. Marks LB, Bentzen SM, Deasy JO, Kong FM, Bradley JD, Vogelius IS, El Naqa I, Hubbs JL, Lebesque JV, Timmerman RD, et al: Radiation dose-volume effects in the lung. Int J Radiat Oncol Biol Phys 76 (Suppl 3): S70-S76, 2010.

24. Kirkpatrick JP, van der Kogel AJ and Schultheiss TE: Radiation dose-volume effects in the spinal cord. Int J Radiat Oncol Biol Phys 76 (Suppl 3): S42-S49, 2010.

25. Trotti A, Colevas AD, Setser A, Rusch V, Jaques D, Budach V, Langer C, Murphy B, Cumberlin R and Coleman CN: CTCAE v3.0: Development of a comprehensive grading system for the adverse effects of cancer treatment. Semin Radiat Oncol 13: 176-181, 2003.

26. Cox JD, Stetz J and Pajak TF: Toxicity criteria of the Radiation Therapy Oncology Group (RTOG) and the European Organization for Research and Treatment of Cancer (EORTC). Int J Radiat Oncol Biol Phys 31: 1341-1346, 1995.

27. Eisenhauer EA, Therasse P, Bogaerts J, Schwartz LH, Sargent D, Ford R, Dancey J, Arbuck S, Gwyther S, Mooney M, et al: New response evaluation criteria in solid tumours: Revised RECIST guideline (version 1.1). Eur J Cancer 45: 228-247, 2009.

28. Ku GY and Ilson DH: Esophagogastric cancer: Targeted agents. Cancer Treat Rev 36: 235-248, 2010.

29. Kordes S, Cats A, Meijer SL and van Laarhoven HW: Targeted therapy for advanced esophagogastric adenocarcinoma. Crit Rev Oncol Hematol 90: 68-76, 2014.

30. Zhang P, Xi M, Zhao L, Shen JX, Li QQ, He LR, Liu SL and Liu MZ: Is there a benefit in receiving concurrent chemoradiotherapy for elderly patients with inoperable thoracic esophageal squamous cell carcinoma? PLoS One 9: e105270, 2014.

31. Xing L, Liang Y, Zhang J, et al: Definitive chemoradiotherapy with capecitabine and cisplatin for elder patients with locally advanced squamous cell esophageal cancer. J Cancer Res Clin Oncol 140: 867-872, 2014.

32. Richards D, Kocs DM, Spira AI, David McCollum A, Diab S, Hecker LI, Cohn A, Zhan F and Asmar L: Results of docetaxel plus oxaliplatin $(\mathrm{DOCOX}) \pm$ cetuximab in patients with metastatic gastric and/or gastroesophageal junction adenocarcinoma: Results of a randomised phase 2 study. Eur J Cancer 49: 2823-2831, 2013

33. Pinto C, Di Fabio F, Siena S, et al: Phase II study of cetuximab in combination with FOLFIRI in patients with untreated advanced gastric or gastroesophageal junction adenocarcinoma (FOLCETUX study). Ann Oncol 18: 510-517, 2007.

34. Pérez-Soler R, Delord JP, Halpern A, Kelly K, Krueger J, Sureda BM, von Pawel J, Temel J, Siena S, Soulières D, et al: HER1/EGFR inhibitor-associated rash: Future directions for management and investigation outcomes from the HER1/EGFR inhibitor rash management forum. Oncologist 10: 345-356, 2005. 